\title{
Preparation and Gas Sensing Properties of Orderly Porous Tin Dioxide
}

\author{
Junliang Liu ${ }^{\mathrm{a}}$, Yiyang Zhang ${ }^{\mathrm{b}}$, Chengwu Zhou ${ }^{\mathrm{a}}$, Haiyan Chen ${ }^{\mathrm{a}, \mathrm{c}}$, Ming Zhang ${ }^{\mathrm{a}}$ \\ ${ }^{a}$ School of Chemistry and Chemical Engineering, Yangzhou University, Yangzhou, PR China, 225002 \\ ${ }^{\mathrm{b}}$ School of System Informatics, Kobe University, Kobe, Japan, 657-8501 \\ c Yangzhou Polytechnic College, Yangzhou, PR China, 225127 \\ *Corresponding Author: 1xyzhangm@yzu.edu.cn
}

\begin{abstract}
The orderly porous tin dioxide materials have been fabricated by a templated method with using mono-dispersed polystyrene microspheres as the templates. The assynthesized porous tin dioxide materials showed a good sensing property with a low optimum working temperature of $310{ }^{\circ} \mathrm{C}$ and a wide sensor response of alcohol, a fast response time of $27 \mathrm{~s}$ and a short recovery time of $31 \mathrm{~s}$ (as the alcohol concentration was $200 \mathrm{ppm}$ ), which was much better than those of the sol-gel derived tin dioxide ultrafine powders.
\end{abstract}

Keywords: Tin dioxide, porous, gas sensing.

\section{Introduction}

Tin dioxide as one kind of wide bandgap semiconductors is characterized of excellent gas sensing properties $^{(1-2)}$, which gives it a broad application in commercially manufactured sensors for toxic and combustible gases. To enhance its sensitivity and decrease its working temperature, the design of porous order structures is believed to be one of the feasible ways ${ }^{(3-7)}$. The designed structures with higher specific surface and porosity could improve sensitivity, and reduce response and recovery times $^{(3-7)}$. Focusing on enhancing gas sensing properties of tin dioxide materials, many efforts have been made and the results are encouraging. In this paper, a polymer templated method has been designed and used to fabricate orderly porous tin dioxide materials, which showed a good alcoholsensing property.

\section{Experimental}

\subsection{Preparation}

The porous tin dioxide was synthesized by a templated method, which was briefly descried as follows: 1) Stannous oxalate as the tin source was dissolved in the citric acid aqueous solution, and then triethanolamine was employed to adjust the $\mathrm{pH}$ value to 6-7. After stirring and reacting for $8 \mathrm{~h}$, a transparent yellow sol was obtained. 2) Mono-dispersed polystyrene micro-spheres with the particle size about 400 $\mathrm{nm}$ were fabricated via a dispersion polymerization ${ }^{(8)}$ and dispersed in the above yellow sol. The mixture was centrifugated and the precursor was obtained. After redispersed in alcohol, the polystyrene microspheres combining with tin sol were assembled under the gravity force with evaporation of alcohol at $35^{\circ} \mathrm{C} .3$ ) the templates with tin sol were transferred into a crucible and place in a muffle furnace. The polystyrene templates were removed after heat-treatment at $500{ }^{\circ} \mathrm{C}$ for $2 \mathrm{~h}$ and the porous tin dioxide powders were collected. For comparison, the conventional tin dioxide powders were fabricated by a solgel method with stannous oxalate as the starting material and citric acid as the chelating agent.

\subsection{Characterization}

The phase compositions of the synthesized tin dioxide powders were identified by the X-ray diffraction measurement with $\mathrm{Cu} \mathrm{K} \alpha$ (Bruker, D8 Advance). Their morphology was analyzed by scanning electronic microscope (Hatachi, S4800). Their gas sensing properties were characterized by a WS-30A gas sensitivity instrument (Zhengzhou Winsen Electronics Co., Ltd., Henan, China). The pastes containing tin dioxide powders were uniformly coated on a ceramic tube with electrodes. Prior to the test, the assembled samples were aged at $300^{\circ} \mathrm{C}$ for about a week. The alcohol was heated to form the gas source, injected into a glass chamber and mixed with ambient air. The gas sensing properties were determined according to the testing principle of gas sensor ${ }^{(3,9)}$. The sensor response $(S)$ was determined by 
the following formula:

$$
\mathrm{S}=R_{a} / R_{g}
$$

Where $R_{\mathrm{g}}$ and $R_{\mathrm{a}}$ were calculated by:

$$
\begin{aligned}
& \mathrm{R}_{\mathrm{g}}=\mathrm{R}_{\mathrm{L}}\left(\mathrm{V}_{\mathrm{c}}-\mathrm{V}_{\mathrm{g}}\right) / \mathrm{V}_{\mathrm{g}} \\
& \mathrm{R}_{\mathrm{a}}=\mathrm{R}_{\mathrm{L}}\left(\mathrm{V}_{\mathrm{c}}-\mathrm{V}_{\mathrm{a}}\right) / \mathrm{V}_{\mathrm{a}}
\end{aligned}
$$

$R_{\mathrm{L}}$ is the load resistance with a certain value, $V_{\mathrm{c}}$ is the supplied circuit voltage. The output voltage $\left(V_{\text {out }}\right)$ reflects the immediate voltage of the load resistor in the entire testing process, and it is described as $V_{\mathrm{a}}$ and $V_{\mathrm{g}}$ when the sensor is loaded in ambient air and alcohol gas.

\section{Results and discussion}

Fig.1 gave the XRD patterns of the as-synthesized porous tin dioxide and sol-gel derived tin powders. All the diffraction peaks were indexed and ascribed to tetragonal rutile tin dioxide and the relative intensities of the diffraction peaks were very similar to JCPDS No. 41-1445, indicating the successful formation of single phase tine dioxide without obvious impurity phases. There was no carbonizing product of polystyrene remaining in the synthesized porous tin oxide. Also, the sol-gel derived powders consisted of single phase tin dioxide.

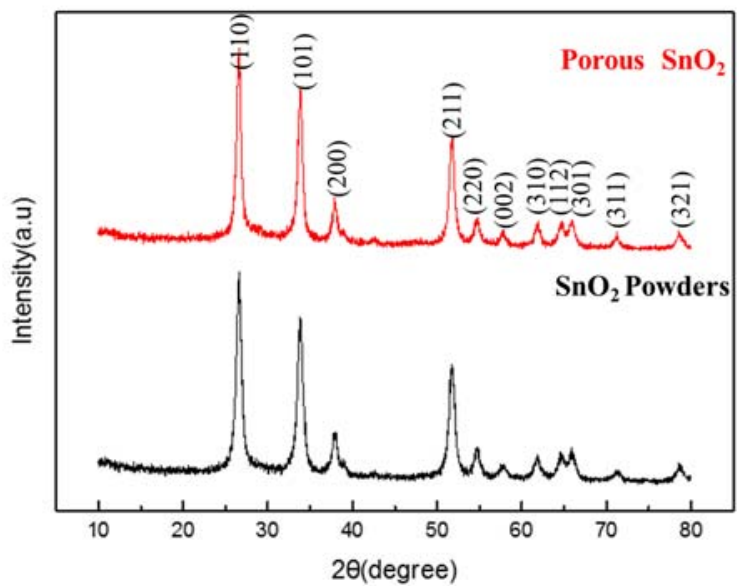

Fig. 1. The XRD patterns of the synthesized porous tin oxide(up) and sol-gel derived powders (down).

Referring to the morphology of the two kinds of tine dioxide. As shown in Fig.2, the as-synthesized tin dioxide via the designed template method demonstrated a porous structure with the homogenous pores of about $200 \mathrm{~nm}$ in it. The pores were of high orderly arrangement owing to the self-assembly of the polystyrene micro-spheres. The thickness of the pore walls was in the nano scale about 40 $\mathrm{nm}$. Such a highly order and pours structure may increase the contacting area between tested gas and $\mathrm{SnO}_{2}$ surface and provide abundant diffusion channels for gas molecules, which was expected to have a good gas sensing property. For comparison, the sol-gel derived tin dioxide ultrafine powders were of a broad size distribution in the range of $200 \mathrm{~nm}$ to 1 $\mu \mathrm{m}$ and with a severe agglomeration.

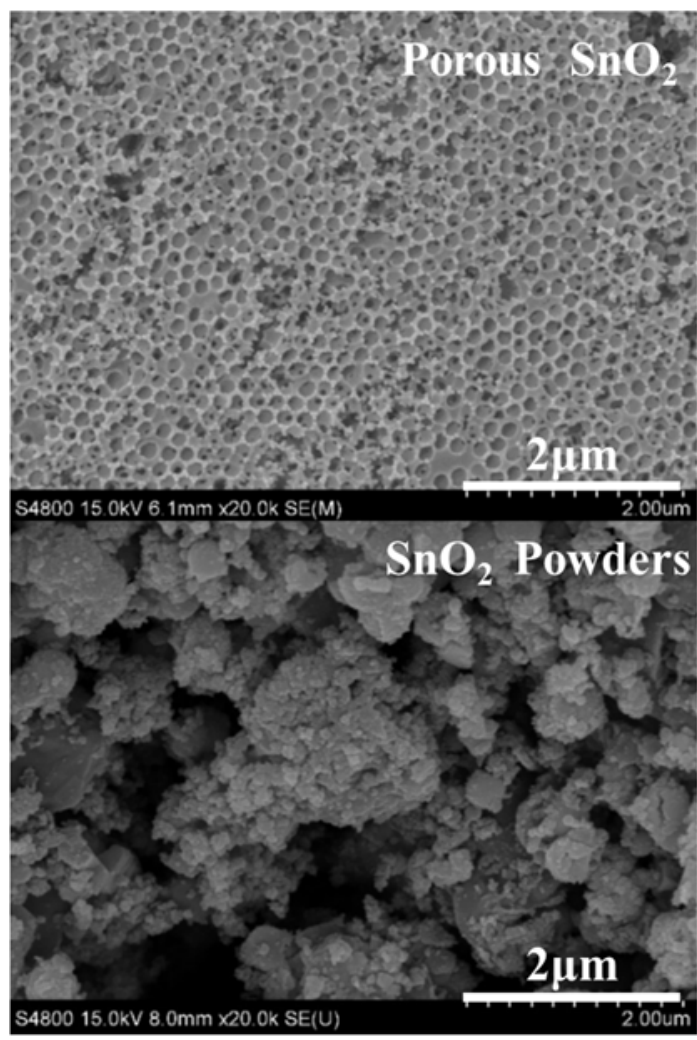

Fig. 2. The SEM images of the synthesized porous tin oxide(up) and sol-gel derived powders (down).

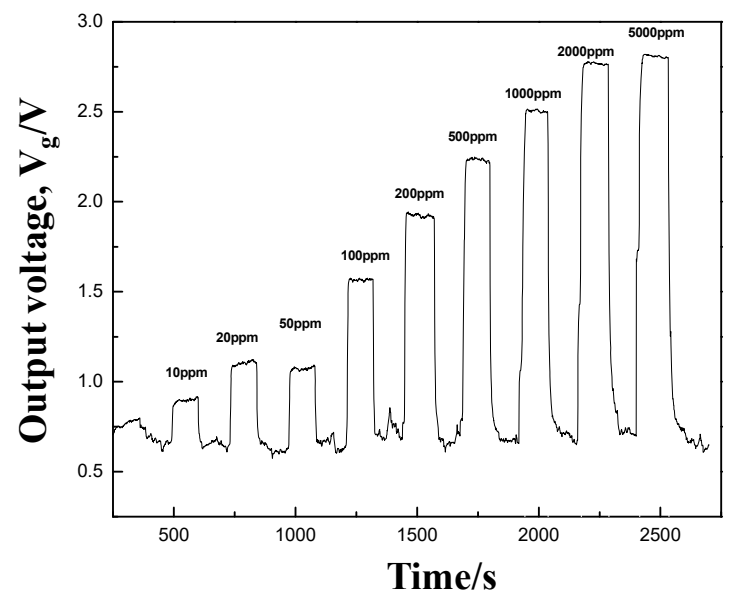

Fig. 3. The output voltage as a function of testing time as loading alcohol gas with various concentrations.

Fig.3 demonstrated the output voltage-testing time curves with increasing alcohol concentration, indicating the gas sensor with the as-synthesized porous tin dioxide approached a stable sensing ability in a wide concentration 
range from 10 to $2000 \mathrm{ppm}$ and the response and recovery time appeared to be very short. The sensor response as a function of alcohol concentration was shown in Fig. 4. As the alcohol concentration increased, the sensor response grew up continuously and approached a near saturation value as the alcohol concentration was over 1000 ppm, which certified it could be utilized as a good gas sensor for alcohol.

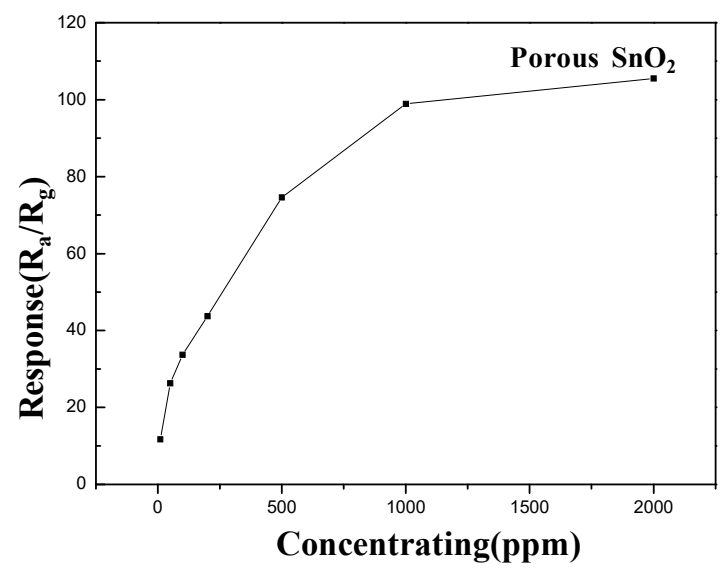

Fig. 4. The sensor response $(S)$ as a function of alcohol concentration.

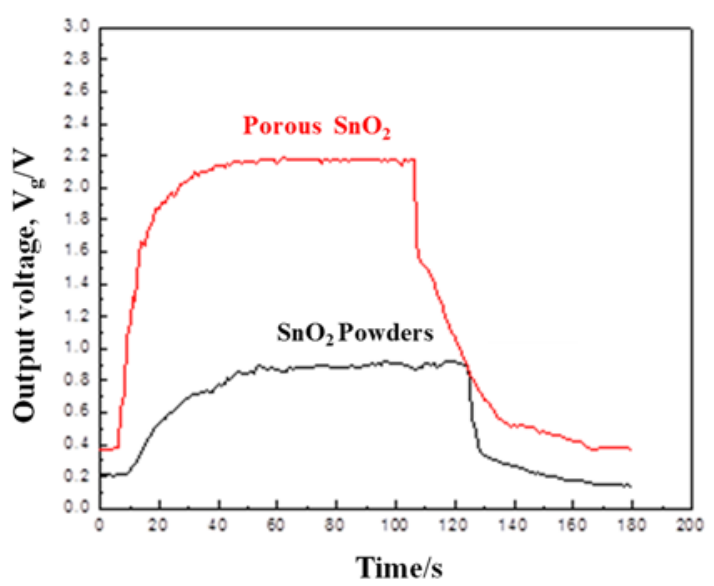

Fig. 5. The output voltage as a function of testing time as loading alcohol gas with concentration of $200 \mathrm{ppm}$.

Fig.5 gave output voltage as a function of testing time as loading alcohol gas with concentration of 200ppm. The gas sensor fabricated by the as-synthesized porous tin dioxide showed a larger sensor response of 43.7, faster response about $27 \mathrm{~s}$ and short recovery time of $31 \mathrm{~s}$, which were much better than those of the gas sensor fabricated by the sol-gel derived tin dioxide (15.2, $33 \mathrm{~s}$ and $35 \mathrm{~s}$, respectively). This was ascribed to the formation of the high orderly and porous structure, which provided a larger contacting area and more abundant diffusion channels.
Moreover, the sensor response-working temperature curves were reflected in Fig.6. It was found that: 1) the gas sensor fabricated by the as-synthesized porous tin dioxide had a higher sensor response all through the working temperature range from 160 to $420{ }^{\circ} \mathrm{C}$, comparing to the gas sensor fabricated by the sol-gel derived tin dioxide. 2) The optimum working temperature decreased from $370{ }^{\circ} \mathrm{C}$ to 310 ${ }^{\circ} \mathrm{C}$ as the successful formation of the high orderly and porous structure.

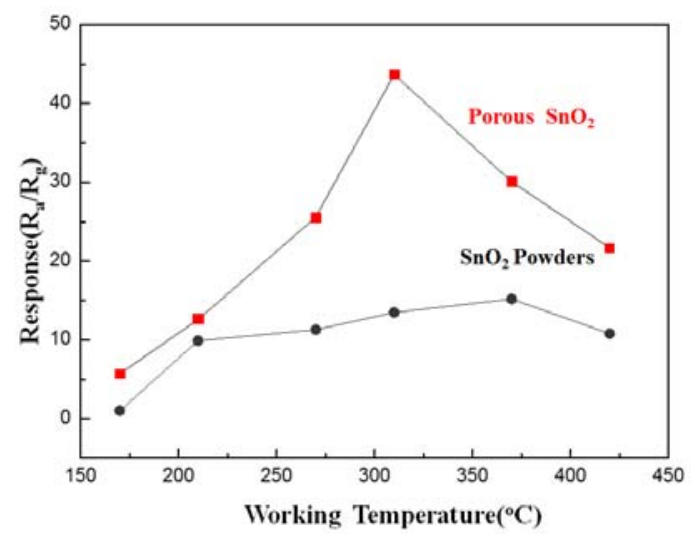

Fig. 6. The sensor response $(S)$ as a function of working temperature.

\section{Conclusions}

In conclusion, high orderly and porous tin dioxide with pore size of $200 \mathrm{~nm}$ and wall thickness of $40 \mathrm{~nm}$ has been successfully fabricated by using the polystyrene microspheres as the templates. The formation of the high orderly and porous structure provided a larger contacting area and more abundant diffusion channels, which endowed the gas sensor with the as-synthesized porous tin dioxide a good sensing property to alcohol: a low optimum working temperature of $310^{\circ} \mathrm{C}$, a wide sensor response of alcohol, and a faster response about $27 \mathrm{~s}$ as well as a short recovery time of $31 \mathrm{~s}$.

\section{Acknowledgment}

The authors acknowledge the financial support from National Natural Science Foundation of China (No.51678292), China Postdoctoral Science Foundation (2016T90512, 2015M570483), the Scholarship of Jiangsu Government for Oversea Study and the Project Funded by the Priority Academic Program Development of Jiangsu Higher Education Institutions (Chemistry). 


\section{References}

(1) N. Yamazoe, N. Miura, "Environmental gas sensing", Sensors and Actuators B, 20, 95-102, 1994.

(2) W. Jin, S. Ma, Z. Tie, W. Li, J. Luo, L. Cheng, X. Xu, T. Wang, X. Jiang, Y. Mao, "Synthesis of hierarchical $\mathrm{SnO}_{2}$ nanoflowers with enhanced acetic acid gas sensing properties", Journal of Applied Surface Science, 353, 71-78, 2015.

(3) Wenjing Du, Nannan Wu, Zhou Wang, Jiurong Liu, Dongmei $\mathrm{Xu}$, Wei Liu, "High response and selectivity of platinum modified tin oxide porous spheres for nitrogen dioxide gas sensing at low temperature", Sensors and Actuators B, 257, 427-435, 2018.

(4) Lee J.-H., "Gas sensors using hierarchical and hollow oxide nanostructures: Overview", Sensors Actuators B: Chemistry, 140, 319-336, 2009.

(5) M.M. Arafat, B. Dinan, S.A. Akbar, A. Haseeb, "Gas sensors based on one dimensional nanostructured metaloxides, a review", Sensors, 12, 7207-7258, 2012.

(6) Hyodo T., Sasahara K., Shimizu Y., Makoto E., "Preparation of macroporous $\mathrm{SnO}_{2}$ films using PMMA microspheres and their sensing properties to $\mathrm{NO}_{\mathrm{x}}$ and $\mathrm{H}_{2}$ ", Sensors Actuators B: Chem., 106, 580-590, 2005.

(7) A. Kolmakov, Y.X. Zhang, G.S. Cheng, M. Moskovits, "Detection of $\mathrm{CO}$ and $\mathrm{O}_{2}$ using tin oxide nanowire sensors", Advanced Materials, 12, 997-1000, 2003.

(8) Zhu Wen, Huang Fang-ting, Yang Run-miao, Zhang Ming, "Preparation of Size Controllable Monodispersed Polystyrene Microspheres by Dispersion Polymerization", Journal of Materials Science and Engineering, 4, 595-599, 2012.)

(9) Jun Zhang, Shurong Wang, Mijuan Xu, Yan Wang, Baolin Zhu, Shoumin Zhang, Weiping Huang and Shihua Wu, "Hierarchically Porous $\mathrm{ZnO}$ Architectures for Gas Sensor Application", Crystal Growth \& Design, 9, 3532-3537, 2009. 